\title{
A Rare Case of Acute Simultaneous Bilateral Quadriceps Tendon Rupture - From Diagnosis to Treatment and Review of the Literature
}

\section{João Brito Barroso*, João Pedro Campos, Andreia Moreira, João Quelhas, José Machado, Pedro Santos, Nuno Camelo Barbosa, Filipa and Santos Jorge}

Department of Orthopedic Surgery, Hospital Pedro Hispano, Matosinhos, Porto, Portugal

*Corresponding Author: João Brito Barroso, Department of Orthopedic Surgery, Hospital Pedro Hispano, Matosinhos, Porto, Portugal.
Received: April 12, 2021

Published: May 11, 2021

(C) All rights are reserved by João Brito

Barroso., et al.

\begin{abstract}
Acute simultaneous bilateral quadriceps tendon ruptures are rare. In the majority of the cases they are related to major trauma in patients with well-estabilished predisposing factors to tendinopathy and rupture. We describe a case of bilateral quadriceps tendon rupture sustained after minimal trauma in a patient with no history of systemic diseases. We discuss the etiology and possible risk factors of acute tendon rupture, its treatment and rehabilitation and report a literature review of bilateral quadriceps tendon ruptures.
\end{abstract}

Keywords: Quadricipital Tendon; Bilateral Rupture; Surgery

\section{Introduction}

Quadriceps femoral muscle is the largest muscle in the human body and the primary knee extensor of the leg. Its rupture is not uncommon, happening mostly in men older than 50 years old: $28,9 \%$ of extensor knee injuries in patients over 50 years old are quadriceps tendon ruptures [1].

On the other hand, Simultaneous Bilateral Quadriceps Tendon Rupture (SBQTR) are extremely rare, with fewer than 100 cases reported in the English Literature [2,3] and mostly associated with chronic diseases, including diabetes mellitus, chronic renal disease, hyperparathyroidism, gout, systemic lupus erythematous, Wilson's disease, obesity, older ages and also correlated with some drugs, as steroids, fluoroquinolones and statins [4-6].

We report a clinical case of a 75 year-old male who presented with SBQTR after falling down during a walk.

\section{Case Report}

A 75 year-old man came to our emergency room sitting in a wheelchair, with complaints of pain in both knees and inability to walk. The symptoms had begun in that day during a regular walking, after a stumble and fall, associated with a popping sound and sudden pain on his right knee, and then a second popping on his contralateral knee when trying to maintain balance and total body weight on the left leg. He declined any chronic knee pain before, and presented no other history of trauma or previous injury.

The patient was retired from work, had an active life, with regular daily walks. He denied any heavy labor or new sports practice in the days before. His body mass index was $26,4 \mathrm{~kg} / \mathrm{m}^{2}$.

Past medical history included a benign prostatic carcinoma, eight years before, treated with radiotherapy and no known sequels. A previous gout crisis, four years before, since then treated with prophylactic alopurinol. The patient denied new crisis, and had uric acid values regularly controlled. The hemogram performed in emergency room confirmed the normal values. Finally, the patient had history of chronic gastritis, treated with Proton Pump Inhibitors (PPIs) - Omeprazol 20 mg per day - for the last 10 years.

No other disease or medication was reported, including drugs associated with tendon rupture as steroids or fluoroquinolones. The patient denied tobacco consumption or alcoholics habits. 
Clinical examination revealed swollen bilateral knee joints, with palpable suprapatelar gap (Image 1) of both quadriceps tendon, associated with tenderness and bilateral deficit of active extension.

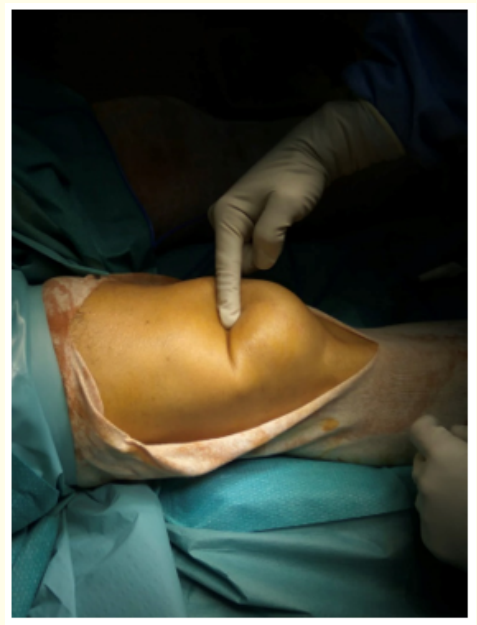

Image 1: Suprapatellar gap sign due to the rupture.

There were no cutaneous injury and neurological and neurovascular examination were normal. X-ray images of knees showed no fracture or bony lesion. Ultrasound images of both knees revealed complete rupture of the quadriceps tendon at patellar insertion on the right knee, and more proximal complete rupture on the left knee.

Three days after his diagnosis, the patient underwent surgical repair of both quadriceps' tendon ruptures. We approached both knees through standard anterior midline incisions. The tendon rupture was exposed, hematoma and fibrous granulation tissue debrided. Both tears were complete tears at the osteotendinous junction. The surgical technique we used was different between sides.

On right side, the tear presented a more distal location (Image 2 and 3) with no remaining tendon tissue on the top of the patella, for which we used a transosseous fixation - the ruptured tendon were trimmed and stitched with no5 FiberWire suture (Arthrex, Naples, Florida), using the Krackow technique. The free ends of the sutures were passed through three drilled holes made from the superior pole to the inferior pole of patella with $2.0 \mathrm{~mm}$ drill bit and tied to reattach the tendon to patella (Image 4 and 5).

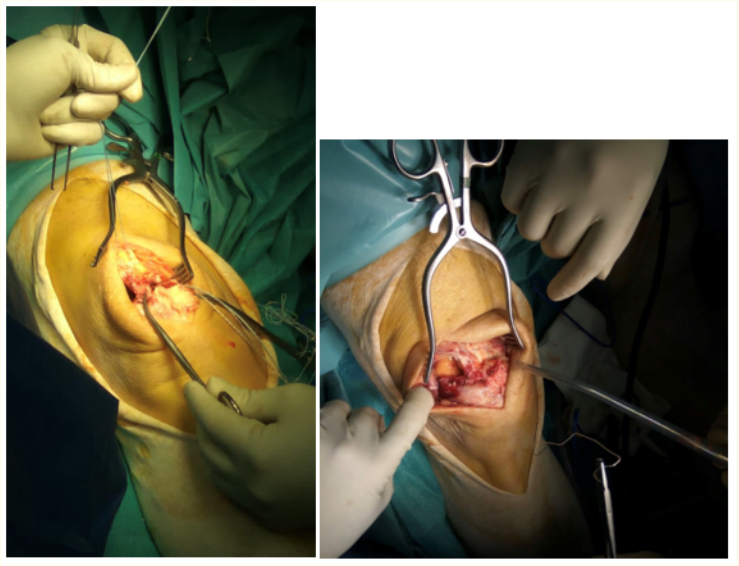

Image 2 and 3: Right knee rupture in the transition tendon-bone (2) and the complete lateral retinaculum tear.
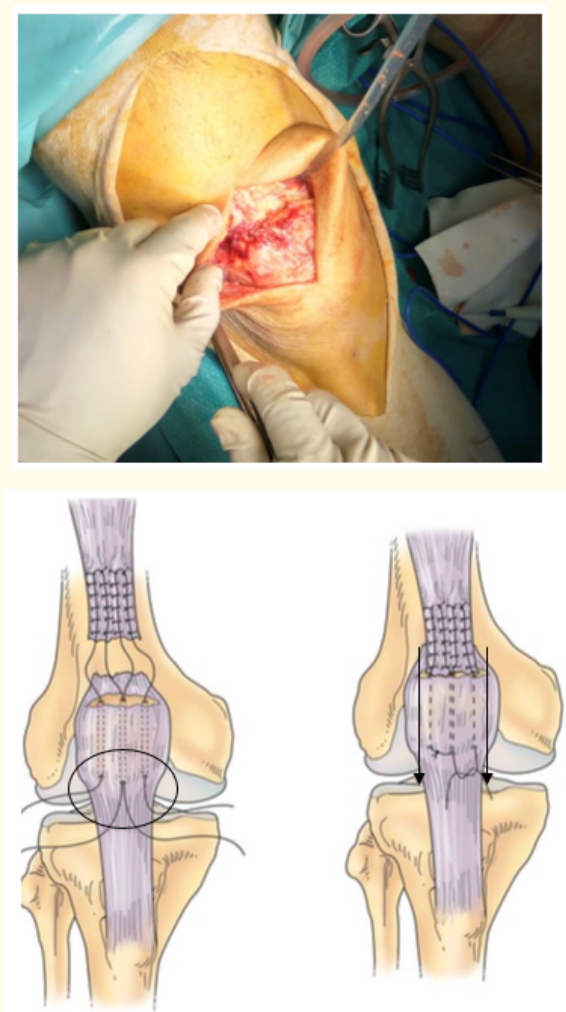

Image 4 and 5: Right tendon after suture with transosseous technique as shown in the image 5 (Transosseous fixation technique used - adapted from www.musculoskeletalkey.com). 
The left side we observed a more proximal tear (midsubstance tear) and in this case we used an end-to-end Krackow suture repair using no5 Ethibond (Johnson \& Johnson, New Brunswick, New Jersey) (Image 6 and 7).

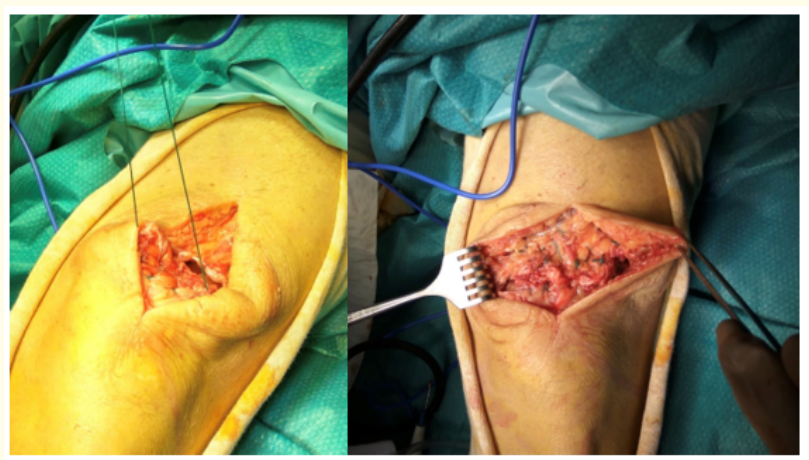

Image 6 and 7: Left tendon rupture in midsubstance of tendon, with end-to-end Krackow sutures repair (Image 7).

We also found a complete retinaculum tear on right side (Image 3 ) and a smaller longitudinal split on the left knee, both repaired with absorbable suture.

Post-surgery both knees were protected with knee immobilizer in full extension.

The patient was advised not to bear weight for two weeks. Afterwards he was allowed to bear weight as tolerated in full extension, with a walker. After four weeks postoperatively, he started physical therapy, with isometric quadriceps exercises, transcutaneous electrical nerve stimulation and flexion from 0 o to $60^{\circ}$. Full motion was allowed after six weeks postoperatively.

The patient presented a positive functional recovery, with bilateral range of motion from $-2^{\circ}$ to $95^{\circ}$ two months post surgery. At six months he presented no pain or effusion, intact straight-leg raise test, $120^{\circ}$ of knee flexion, $5 / 5$ knee extension strength bilaterally and unimpaired gait.

At 12 months after surgery, the patient presented pain free mobility, intact straight-leg raise, range of motion from $-4^{\circ}$ to $125^{\circ}$ and 5/5 knee extension strength bilateral.
Though different surgical technique sutures were used, no differences in the outcome were found between knees.

\section{Discussion and Conclusion}

The quadriceps tendon is the largest tendon of the human body, making the distal attachment of the main extensor muscle - the quadriceps - thus essential to the normal extension of the leg.

Although their unilateral ruptures are common, especially in men over 50 years old [7], SBQTR is a rare pathology, with few cases described [8].

Rupture of the quadriceps tendon is often post-traumatic, mainly when trauma occurs at the moment of the knee is in a semi flexed position or in eccentric contraction as balancing the body to prevent falling [9].

Apart from this typical lesion mechanism, some comorbidities are well established and well-recognized in the bilateral tendon ruptures as predisposing factors. Shah reviewed 55 case reports and concluded that $76 \%$ of patients had medical comorbidities associated as chronic renal disease, hyperparathyroidism, gout, systemic lupus erythematosus, diabetes mellitus, obesity, and prescription drug use as fluoroquinolones, statins and steroids [10].

We find our case of particular interest for various reasons. Firstly, our patient had no evident predisposing risk factors, except for the age and a previous gout crisis episode four years before, since then being well controlled, with uricemia levels in a normal range, controlled annually by the Family Doctor and confirmed in our emergency department with blood analysis.

The patient was not obese and, blood glucose, cholesterol and lipide levels were in normal range.

The long-term PPIs use and its potential association as a risk factor to SBQTR has never been reported before in the literature, to our best knowledge [11]. Although we considered it, it's difficult to establish that causality, as PPIs are among the most prescribed drugs in this age range, and a higher prevalence of similar cases would be expectable. However, for future cases, we would be looking for this class of medication.

Despite this lack of known major risk factors for rupture, we believe the aging and underlaying tendon degeneration, combined 
with possible overuse due to an active lifestyle for this age range are possible factors that precipitated the bilateral ruptures.

Another interest fact was the different localization of the ruptures. This leads us to gather that if the myotendinous junction the interface between muscle and tendon - is well known to be the weakest link in the muscle-tendon chain, the tendinous insertion in the patella pole can also be a susceptible and fragile area, making it possible to have SBQTR in distinct areas of the tendon.

As for the surgical technique, numerous surgical methods have been proposed, but without differences regards to clinical outcomes and complications [12]. We used 2 different sutures techniques, according to the size, location and nature of the tendon rupture.

Both were successful, with no functional differences noted.

A variety of reinforcement techniques - from wire reinforcement to the use of Fascia Lata graft - have been described, to obtain extra-support and stabilization of the primary repair [13,14]. We argue that these techniques are useful and play an important role in cases of poor quality tendon tissue where we can't perform a stable and functional primary repair with the sutures, and in cases of retraction of torn ends - as in the chronic cases or acute but delayed reconstruction procedure.

We support that the surgical technique and the need of reinforcement should be chosen accordingly to the nature, location and time of the tendon rupture.

\section{Bibliography}

1. MR Garner., et al. "Extensor mechanism injuries of the knee: demographic characteristics and comorbidities from a review of 726 patient records". The Journal of Bone and Joint Surgery American Volume 97.19 (2015): 1592-1596.

2. Steiner C and Plamer LH. "Simultaneous bilateral rupture of the quadriceps tendon". American Journal of Surgery 78.5 (1949): 752-755.

3. Ellanti P., et al. "Bilateral synchronous rupture of the quadriceps tendon". Irish Journal of Medical Science 181 (2012): 423425.

4. Lotem M., et al. "Spontaneous rupture of the quadríceps tendo in patients on chronic haemodialysis". Annals of the Rheumatic Diseases 33.5 (1974): 428-429.
5. Celik EC., et al. "Simultaneous and spontaneous bilateral quadríceps tendon rupture". American Journal of Physical Medicine and Rehabilitation 91.7 (2012): 631-634.

6. Stern R and Harwin F. "Spontaneous and simultaneous rupture of both quadriceps tendons". Clinical Orthopaedics and Related Research 147 (1980): 188-189.

7. Katz T., et al. "Bilateral simultaneous rupture of the quadríceps tendon in adult amateur tennis player". Journal of Clinical Rheumatology 12.1 (2006): 32-33.

8. Neubauer T., et al. "Bilateral, simultaneous rupture of the quadriceps tendon: a diagnostic pitfall? Report of three cases and meta-analysis of the literature". Knee Surgery, Sports Traumatology, Arthroscopy 15.1 (2007): 43-53.

9. O'Sheaa K., et al. "Outcomes following quadriceps tendon ruptures”. Injury 33.3 (2002): 257-260.

10. Shah MK. "Outcomes in bilateral and simultaneous quadriceps tendon rupture". Orthopedics 26.8 (2003): 797-798.

11. Brisebois S., et al. "Proton pump inhibitors: Review of reported risks and controversies". Laryngoscope Investigative Otolaryngology 3.6 (2018): 457-462.

12. Ciriello V., et al. "Clinical outcomes after repair of quadriceps tendon rupture: a systematic review". Injury 43.11 (2012): 1931-1938.

13. Helito C., et al. "Reinforcement with fascia lata as an alternative in the repair of chronic quadriceps tendon injuries". Upsala Journal of Medical Sciences 119.4 (2014): 354-356.

14. McCormick F., et al. "Autologous hamstring tendon used for revision of quadiceps tendon tears". Orthopedics 36.4 (2013): e529-e532.

\section{Volume 4 Issue 6 June 2021 (C) All rights are reserved by João Brito Barroso., et al.}

